\title{
The analysis of the accuracy of spatial models using photogrammetric software: Agisoft Photoscan and Pix4D
}

\author{
Adrianna Barbasiewicz ${ }^{1}$, Tadeusz Widerski ${ }^{1, *}$, and Karol Daliga ${ }^{1}$ \\ ${ }^{1}$ Gdansk University of Technology, Faculty of Civil and Environmental Engineering, Gdansk, Poland
}

\begin{abstract}
This article was created as a result of research conducted within the master thesis. The purpose of the measurements was to analyze the accuracy of the positioning of points by computer programs. Selected software was a specialized computer software dedicated to photogrammetric work. For comparative purposes it was decided to use tools with similar functionality. As the basic parameters that affect the results selected the resolution of the photos on which the key points were searched. In order to determine the location of the determined points, it was decided to follow the photogrammetric resection rule. In order to automate the measurement, the measurement session planning was omitted. The coordinates of the points collected by the tachymetric measure were used as a reference system. The resulting deviations and linear displacements oscillate in millimeters. The visual aspects of the cloud points have also been briefly analyzed.
\end{abstract}

\section{Introduction}

Photogrammetric measurements are increasingly becoming an alternative to traditional geodetic measurements. This is because they are faster and easier to perform, and the results are received almost immediately. There are many photogrammetric software, but it is hard to tell from the user panel which of them have more accurate measurement results. Estimation of possible uncertainties generated by software is very important for further analysis of measurement results. Also knowledge of distribution of possible errors is important because we know if we can freely use mean values or not.

In this article were decided to compare two popular photogrammetric software: Agisoft Photoscan and Pix4D.

\section{The collection of measurement data}

For collecting photos were used the Canon 5D digital camera. Together 126 images were obtained.

\section{Used software}

Agisoft Photoscan and Pix4D were used for the photogrammetric designation of points. Both programs use still images of stationary objects to generate point clouds, 3D models and orthophotomaps. They are widely used in aviation and engineering photogrammetry, computer animations and remote sensing. With the ability to export processed data to a variety of formats, they are compatible with graphical programs (such as
Autocad) or GIS analysis software (such as ArcGis). These programs also have similar hardware and functionality requirements.

\section{Development of measurement data}

Determining coordinates of the points defined in both programs was similar. First, the pictures were imported into the programs and then merged. Pix 4D uses the Initial Processing tool and Agisoft Photoscan - Align Photos. In both cases, it was possible to set the image size to which the similarity points (characteristic, base) were searched. Three levels of accuracy were selected: - low - $1 / 4$ resolution is taken into account,

- medium - the resolution of $1 / 2$ is taken into account, - high - a full resolution photo is taken into account.

These functions pre-determine the parameters of the internal and external orientation of the images. As a result, clouds of points without spatial reference were obtained. In order to give georeference, the coordinates of three points of photogrammetric matrix were imported. Minimum three points with known coordinates are required by photogrammetric retroreflection rule. In both programs, exactly the same points were selected and marked exactly on the same location on images. Using Create Markers (Agisoft) and GCP (Ground Control Points) / MTP (Manual Tie Points) Manager (Pix4D), 21 marked points were created and marked on the photos in their actual location. The final stage of the point determination procedure was to optimize the internal and external orientation parameters of the images. In this way coordinates of the point were obtained.

\footnotetext{
${ }^{*}$ Corresponding author: tadeusz.widerski@.pg.edu.pl
} 


\section{Analysis of results}

The analysis concerned:

- analysis of calibration parameters,

- determination and analysis of angular and linear displacements,

- visual analysis of obtained images.

\subsection{Analysis of calibration parameters}

The calibration parameters were different from one another depending on the program used to generate them. There were differences in the location of the main point. The reason for this was the different origin of pixel frame. Agisoft Photoscan determines the location of the points (designated and main) based on the origin of the coordinate system located in the upper left corner of the image. Pix4D takes the lower left corner of the photo as the starting point. Therefore, the focal lengths are different. The biggest difference between the focal lengths, of the order of 4.2386 pixels, was set for the image combining with medium accuracy. It has also been noted that the Agisoft Photoscan program determines four coefficients of radial and tangential distortion, while the Pix4D program determines three coefficients of radial distortion and two tangential distances. In addition, Agisoft's program determines two non-orthogonal affine coefficients. In general, the calculations in both programs are similar. However, there are three major differences between them:

- Determination of distortion corrections using different developments of the Brown model,

- Agisoft Photoscan, when calculating the focal point, takes into account affine deformations,

- Pix4D has already calculated the coordinates of the main point position while Agisoft Photoscan uses a focal offset and adds half the width and length of the image each time.

\subsection{Determination and analysis of angular and linear displacements}

The next step in the analysis was to determine the values of linear and angular displacements between the coordinates of points determined during the tachymetric measurement and the coordinates determined by the photogrammetric method. During the calculation, it was noted that the Pix4D program did not assign a position of the 12 points determined at the lowest accuracy settings. The reason of that situation was lack of external orientation for 37 images. Therefore, it was decided to skip a comparative analysis of the determined point coordinates obtained from lowest accuracy parametersAdditionally, it was noted that the Pix4D software treats photopoints as fixed points and location values were not estimated (as opposed to Agisoft Photoscan). For linear and angular displacement determined at medium and high accuracy levels, histograms were made and their indices were calculated.

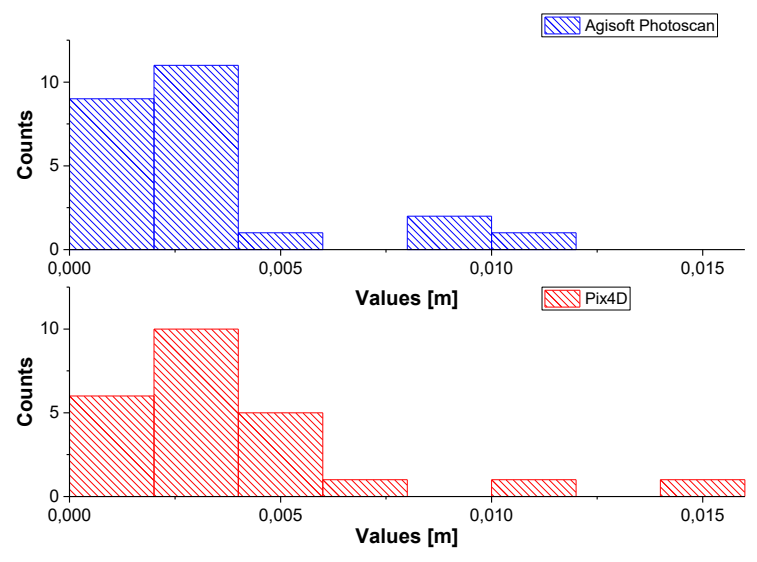

Fig. 1. Linear displacement. Accuracy: medium.

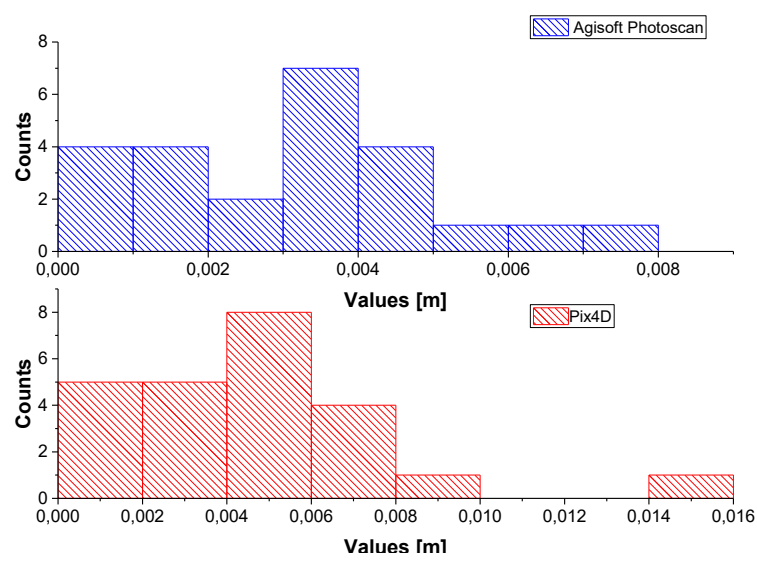

Fig. 2. Linear displacement. Accuracy: high.

Figs. 1 and 2 show histograms of linear displacement values for medium and high accuracy. By comparing the histograms, it can be seen that only histogram made for Agisoft's medium accuracy has a normal distribution. For this reason, the range of $+/-2 \sigma$ were used to analyze the coordinate difference vectors, which is around $95 \%$ of the observed values. For this range, the accuracy of the tachimeter is also given. Linear displacements for the remaining accuracy have a right-skewed distribution. This means that the mean and standard deviation are not reliable indicators used in the analysis. Instead, it was decided to use the median and quanta values. In each of the analyzed cases, the $95 \%$ interval is quantified. In Table 1, median values and quantiles for the $95 \%$ stretch are included.

Table 1. Indicators of linear displacement.

\begin{tabular}{|l|l|l|l|}
\hline & \multicolumn{3}{|c|}{ Agisoft Photoscan } \\
\hline & LOW & MEDIUM & HIGH \\
\hline $\mathrm{R}_{\mathrm{S}}[\mathrm{m}]$ & 0.00551 & 0.00321 & 0.00314 \\
\hline $\mathrm{R}_{\mathrm{med}}[\mathrm{m}]$ & 0.00461 & 0.00337 & 0.00252 \\
\hline $\mathrm{P}_{1}[\mathrm{~m}]$ & - & -0.00069 & - \\
\hline$\sigma[\mathrm{m}]$ & 0.00575 & 0.00195 & 0.00272 \\
\hline $\mathrm{P}_{2}[\mathrm{~m}]$ & - & 0.00711 & - \\
\hline $\mathrm{Q}_{2,5}[\mathrm{~m}]$ & 0.00052 & - & 0.00038 \\
\hline
\end{tabular}




\begin{tabular}{|l|l|l|l|}
\hline $\mathrm{Q}_{97,5}[\mathrm{~m}]$ & 0.02797 & - & 0.01017 \\
\hline & \multicolumn{3}{|c|}{ Pix4D } \\
\hline $\mathrm{R}_{\mathrm{S}}[\mathrm{m}]$ & 0.00666 & 0.00448 & 0.00375 \\
\hline $\mathrm{R}_{\mathrm{med}}[\mathrm{m}]$ & 0.00508 & 0.00434 & 0.00287 \\
\hline $\mathrm{P}_{1}[\mathrm{~m}]$ & - & - & - \\
\hline$\sigma[\mathrm{m}]$ & 0.00793 & 0.00319 & 0.00342 \\
\hline $\mathrm{P}_{2}[\mathrm{~m}]$ & - & - & - \\
\hline $\mathrm{Q}_{2,5}[\mathrm{~m}]$ & 0.00000 & 0.00000 & 0.00000 \\
\hline $\mathrm{Q}_{97,5}[\mathrm{~m}]$ & 0.02452 & 0.01415 & 0.01429 \\
\hline
\end{tabular}

where:

$\mathrm{R}_{\mathrm{S}}$ - average value of offset vector length,

$\mathrm{R}_{\text {med }}$ - median length of offset vector,

$\sigma$ - standard deviation,

$\mathrm{P}_{1}, \mathrm{P}_{2}$ - limit values for $95 \%$ range for medium accuracy (Agisoft),

$\mathrm{Q}_{2.5}, \mathrm{Q}_{97.5}$ - lower and upper interquantile spacing values for the $95 \%$ range.

It was noted that for normal distribution the median value is slightly higher than the mean. In other cases, this dependency is reversed. This is confirmed on the rightskewed side of the slope for the remaining accuracy. It is worth to mention that the right-skewed distribution is not the wrong result. It informs that most of observed values fall below their mean values. For each considered accuracy with right-skewed distribution, all observed values fall within the $95 \%$ range. Thus the $\mathrm{Q}_{2.5}$ and $\mathrm{Q}_{97.5}$ quintiles are respectively the minimum and maximum values.

To investigate angular shifts, histograms for the azimuthal angles (Fig. 3, Fig. 4) and the horizontal angles (Fig. 5, Fig. 6) were created. At histograms, unit of horizontal axis was marked as $G$ which means, in this situation, grads. Full angle measured in grads is equal 400 grads. Azimuthal angles describes directions of horizontal coordinates changes. This changes were calculated by taking into account measured point coordinates and coordinates estimated by software.
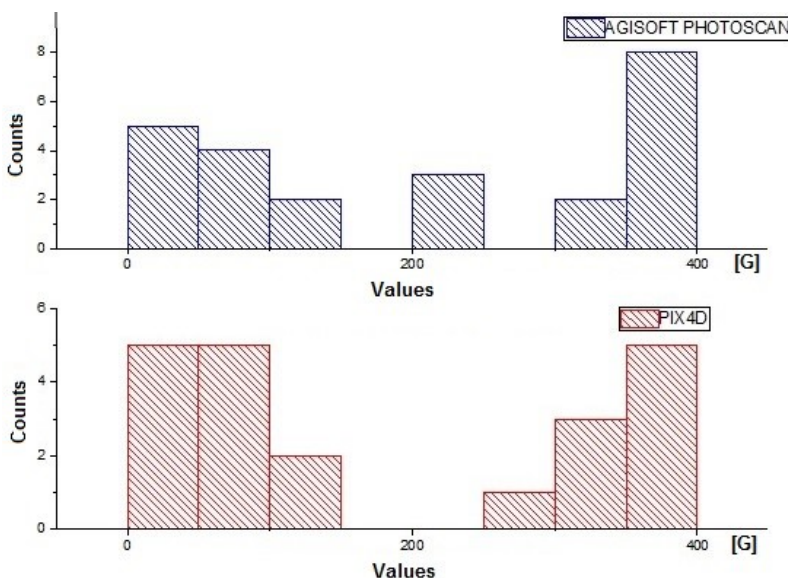

Fig. 3. Histograms of the azimuthal angles. Accuracy: medium.

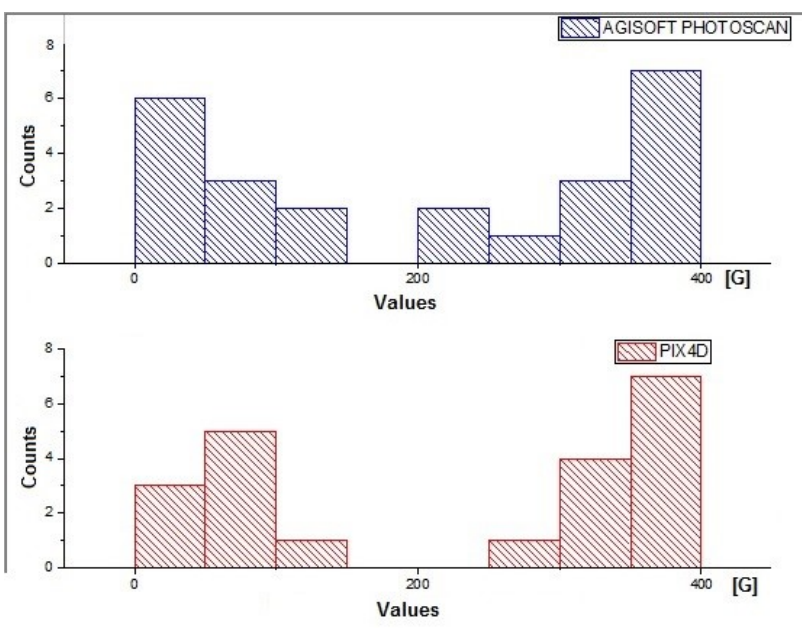

Fig. 4. Histograms of the azimuthal angles. Accuracy: high.
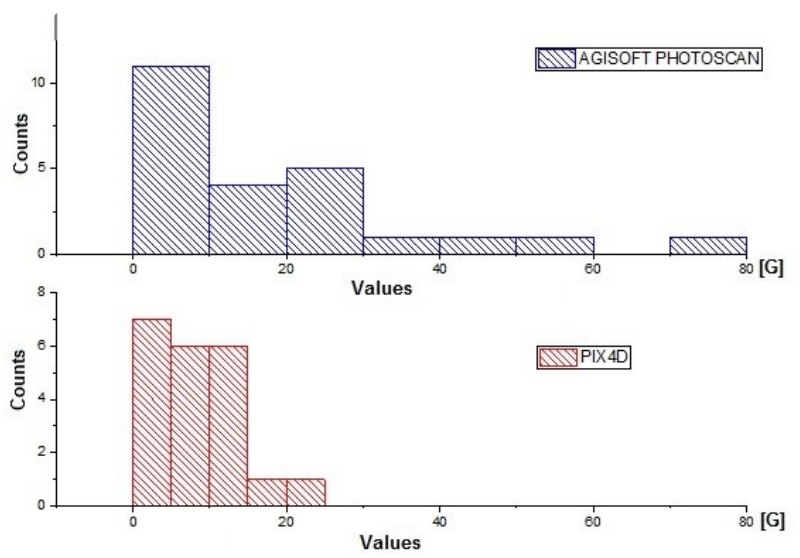

Fig. 5. Histograms of the horizontal angles. Accuracy: medium.

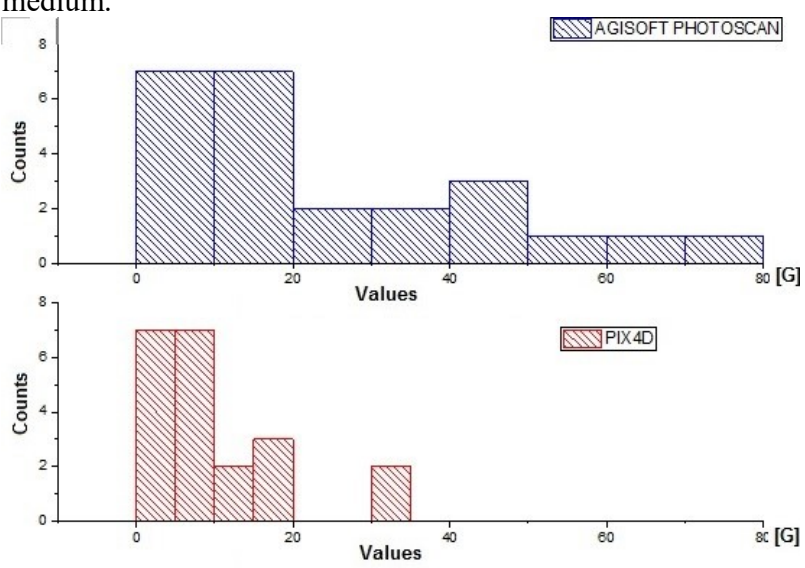

Fig. 6. Histograms of the horizontal angles. Accuracy: high.

On the histograms of the azimuthal angles, bimodal distributions can be observed. The highest incidence rates are between 0 and 100 grads and 300 and 400 grads. This means that the largest azimuthal deviations occur between the north-west and the northeast directions.

The horizontal deviation values for Agisoft Photoscan have a right-skewed distribution. The same accuracy was noted on the histogram for high accuracy in Pix4D. Only the values of the angles determined on the medium accuracy on Pix4D have a normal 
distribution. On all histograms, the highest incidence was observed for angles of up to 20 grads. Agisoft Photoscan had set points where the horizontal angular deviation occurs between 0 and 90 grads. For Pix4D, the maximum limit of the compartment was 50 grads.

\subsection{Visual analysis of obtained images}

Visual analysis focused on the appearance of point clouds and on the number of points generated. It was found that the thickest point cloud was created on the medium accuracy in both computer programs. To confirm this judgment, the exact number of points generated has been verified. On the medium level of accuracy, Agisoft Photoscan created a point cloud composed of 36425 points; on the highest level the point cloud contained 33543 points. Unfortunately, it was not possible to calculate the number of points forming a point cloud in Pix4D, but visual impressions confirm the dependency observed in the first program. In both programs on the high levels of accuracy was a noticeable improvement in the smoothing of points and their concentration. Figs. from 7 to 9 show the appearance of the created point clouds.
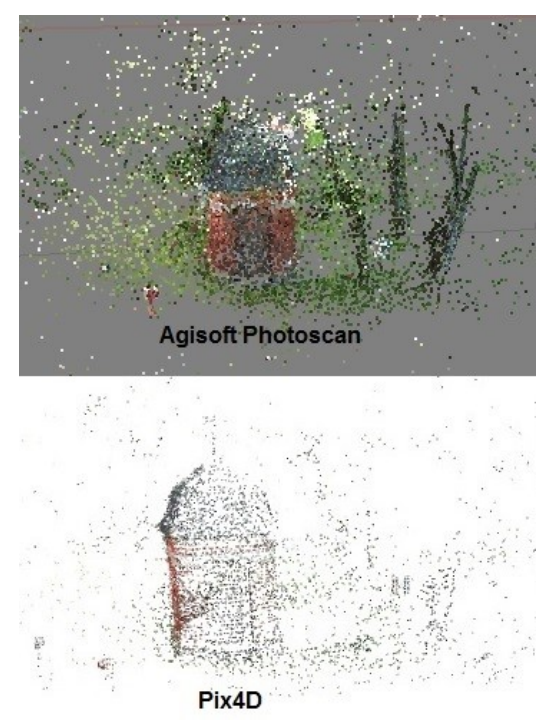

Fig. 7. Point clouds. Accuracy: low.

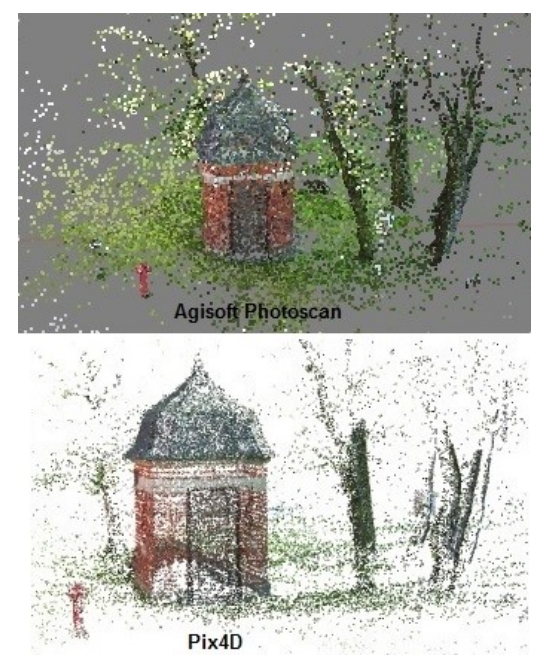

Fig. 8. Point clouds. Accuracy: medium.

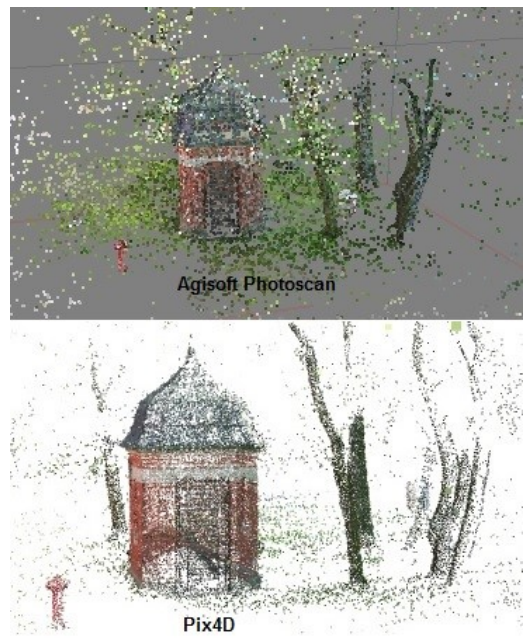

Fig. 9. Point clouds. Accuracy: high.

\section{Summary}

For both programs received deviations were millimeters. Most of the observed values of angular and linear displacement are within the accuracy range of $95 \%$. Only the points marked on the images between which a small convergent angle has been maintained, exceeds the set interval.

It has been noted that deviations and angular-linear displacements for medium and high accuracy in both programs are similar. Thanks to this observation, it can be concluded that calculations performed on medium accuracy are also reliable as calculations performed on the high accuracy. Moreover, calculations performed for medium accuracy were faster than performed for high accuracy. It is important when user doesn't have highend computed and needs good quality of development.

The largest differences in positioning were observed at the lowest accuracy level. Pix4D has not set the position of 12 points determined.

On the basis of the analysis, it was found that satisfactory results can be obtained without the need to mark all photopoints during 3D photogrammetric studies and without exact planning a measurement session. To confirm this thesis additional measurements should be made to prove the reproducibility of the measurement. 


\section{References}

1. Agisoft LLC, Agisoft PhotoScan User Manual: Professional Edition, Version 1.2 (2016)

2. J. Bernasik, S. Mikrut, Fotogrametria Inżynieryjna (Kraków, 2007)

3. M. Korumaz, M. Betti, A. Conti, G. Tucci, G. Bartoli, V. Bonora, A. G. Korumaz, L. Fiorini, Engineering Structures 153, 224 (2017)

4. Z. Kurczyński, Fotogrametria (Wydawnictwo Naukowe PWN SA, Warszawa, 2014)

5. J. McCarthy, Journal of Archaeological Science 43, 175 (2014)

6. J. A. Morgan, D. J. Brogan, P. A. Nelson, Geomorphology 276, 125 (2017)

7. Pix4D SA, Pix4Dmapper 3.2 User Manual (2017)

8. R. Tokarczyk, I. Stanios, Przegląd geodezyjny 76 (6), 7 (2004)

9. Z. Sitek, Fotogrametria ogólna i inżynieryjna (PPWK, Warszawa, 1990)

10. A. Wróbel, Zarys Fotogrametrii (http://home.agh.edu.pl/ awrobel/resources/Zarys\% 20fotogrametrii.pdf, access: 2017) 